\title{
A linha ténue entre a demência e depressão no idoso: relato de caso
}

Joana Pinto Carneiro, * Helena Cabral**

\section{RESUMO}

Introdução: Os problemas de memória em idosos são um motivo frequente de consulta em cuidados de saúde primários. Através desta queixa comum surgem desfechos clínicos distintos, importando a sua identificação precoce e orientação atempada. Segue-se um caso clínico ilustrativo de como abordar este motivo de consulta em cuidados de saúde primários, quais os potenciais diagnósticos, vertentes terapêuticas e respetivas especificidades no doente idoso.

Descrição do caso: Mulher, de 82 anos, viúva há dois anos, pertencente a uma família alargada. A utente vem à consulta trazida pela filha, sua cuidadora informal. A familiar refere que a mãe parece apática, triste e mais sonolenta nos últimos meses. Relata ainda que, de forma progressiva, deixou de participar nas tarefas de casa, não quer sair, evidenciando recorrentemente um discurso repetitivo e arrastado. Mais recentemente apresenta lapsos de memória episódicos e lentidão psicomotora. No decorrer da consulta são ilustrados alguns episódios do quotidiano, designadamente dificuldade crescente nos trocos da mercearia, em reconhecer nomes de "conhecidos" e de determinados objetos, apresentando ainda episódios recorrentes de desorientação espacial ("perde-se por diversas vezes na via pública"). Foram colocadas como possíveis hipóteses de diagnóstico: quadro depressivo com declínio cognitivo associado ou processo demencial inicial com sintomas depressivos. A doente inicia prova terapêutica com sertralina, demonstrando melhoria significativa do humor, do desempenho funcional e avaliação cognitiva sem alterações.

Comentário: A depressão não é uma consequência natural do envelhecimento. A maioria dos idosos que recorre aos cuidados de saúde primários prioriza as queixas somáticas em detrimento do sentimento de tristeza que facilmente é reprimido. Por outro lado, poder-se-á estar a tratar pseudodemências em virtude de alterações cognitivas, potencialmente reversíveis com tratamento antidepressivo/psicoterapia.

Palavras-chave: Demência; Défice Cognitivo; Depressão; Idoso.

\section{INTRODUÇÃO}

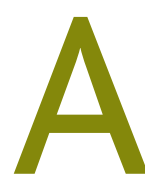

memória é uma das faculdades mentais, cuja alteração mais motiva o idoso a recorrer ao seu médico de família. Embora este declínio possa decorrer dos processos fisiológicos próprios do envelhecimento, é importante reconhecê-lo como possível estágio de transição para demência ou indício de patologia do foro depressivo.

Em termos mundiais, sabe-se que o defeito cognitivo e a demência são situações de elevada prevalência. ${ }^{1}$ Em 2012, e com base no relatório da Organização Mundial da Saúde e da Associação Alzheimer Disease Inter-

*Médica Interna de Medicina Geral e Familiar. USF Garcia de Orta, ACeS Porto Ocidental

**Médica Assistente de Medicina Geral e Familiar. USF Alfena, ACeS Maia/Valongo national (ADI), a prevalência de demência estimada ao nível mundial foi de $4,7 \%$ em pessoas com 60 ou mais anos, correspondendo a cerca de 35,6 milhões de pessoas. $^{2}$ Atualmente, a previsão é de que cerca de 47,47 milhões sejam portadores de demência, podendo atingir, segundo estimativas recentes, 75,63 milhões em $2030 \mathrm{e}$ 135,46 milhões em 2050. ${ }^{3}$

Em Portugal não existem estudos epidemiológicos suficientes no sentido de identificar a real prevalência destas entidades clínicas no idoso. De acordo com alguns dos estudos nacionais, sabe-se que o defeito cognitivo ligeiro atinge cerca de $12,3 \%$ dos portugueses, sendo que a demência está presente em $2,7 \%$ dos indivíduos entre os 55 e os 79 anos de idade, estimativa que será certamente superior nos mais idosos. ${ }^{1}$ No que 
respeita à depressão na terceira idade, estudos internacionais indicam estar presente em cerca de $10 \%$ da comunidade geriátrica e em $40 \%$ dos idosos internados nos hospitais, unidades de cuidados continuados e residências sénior. ${ }^{4}$

É neste contexto que o médico de família assume uma posição privilegiada, podendo identificar os primeiros sinais/sintomas, permitindo uma intervenção precoce e orientação atempada no que respeita ao diagnóstico, terapêutica, dinâmica familiar e suporte social.

\section{DESCRIÇÃO DE CASO}

Trata-se de uma mulher de 82 anos, viúva há dois anos, pertencente a uma família alargada, Grafar II, com seis anos de escolaridade, doméstica. A utente vem à consulta trazida pela filha, sua cuidadora informal. A familiar refere que a mãe parece apática, triste e mais sonolenta nos últimos seis meses. Relata ainda que, de forma progressiva, deixou de participar nas tarefas de casa (cozinhar, arrumar, costurar), não quer sair (algo que habitualmente fazia com agrado), evidenciando recorrentemente um discurso repetitivo e arrastado. Nos últimos três meses apresenta também lapsos de memória episódicos e lentidão psicomotora. No decorrer da consulta são ilustrados alguns episódios do quotidiano em que se constata considerável limitação funcional, designadamente dificuldade crescente nos trocos da mercearia, em reconhecer nomes de "conhecidos" e determinados objetos, apresentando ainda episódios recorrentes de desorientação espacial ("perde-se por diversas vezes na via pública"). A filha reforça que a mãe sempre se mostrou muito ativa no meio social e familiar, encontrando-se muito preocupada com o atual estado da progenitora, razão pela qual solicitou antecipação da consulta programada.

À data da consulta, a paciente detinha discurso escasso, respondendo apenas ao que lhe era questionado. Foi ainda averiguada alteração do padrão do sono, assinalando-se, na perspetiva da família, maior sonolência diurna (que a doente relativiza) e insónia intermédia. Sublinha-se ainda a desvalorização de todo o quadro sintomático por parte da paciente ao longo da consulta, dizendo tratar-se do "normal para a idade".

Ao exame objetivo apresentava-se corada, hidratada, apirética e normotensa. Não se identificaram alterações relevantes à restante observação física, particularmente ao nível neurológico, tendo-se avaliado os nervos cranianos, a força e tónus muscular, reflexos osteotendinosos, coordenação motora (realizando prova dedo-nariz, movimentos alternantes rápidos, prova de Romberg), sensibilidades e sinais de irritação meníngea. Destaca-se, no entanto, a hipomimia (ausência de expressão facial) e ainda ligeira perda ponderal $(3 \mathrm{Kg})$, sendo percecionado pela cuidadora a diminuição do apetite.

Recorrendo às escalas de avaliação geriátrica, a paciente apresentou os seguintes achados: Mini Mental State Examination ${ }^{5}$ com 21 pontos, correspondente a provável défice cognitivo (Figura 1); Prova Relógio Normal (9 pontos) e Escala de Depressão Geriátrica com evidência de humor depressivo moderado (8 pontos). Foi ainda excluída ideação suicida, com recurso ao questionário de Ideação Suicida de Reynolds (QIS). Fez-se também revisão da medicação em curso, não se identificando fármacos com potencial prejuízo na esfera cognitiva (nomeadamente, benzodiazepinas, betabloqueadores, analgésicos opiáceos, neurolépticos, etc.).

\section{Problemas ativos de saúde}

Hipertensão arterial controlada, dislipidemia e patologia osteoarticular do tipo degenerativo (osteoartrose severa da anca e joelhos).

\section{Medicação habitual}

Losartan 50mg id, sinvastatina $20 \mathrm{mg}$ id e cloridrato de glucosamina 1250mg id. De acrescentar ainda o uso de anti-inflamatório aquando de dor articular, em SOS. Apresentava um índice de Barthel sem alterações de relevo (90 - independente). No que respeita aos seus antecedentes pessoais não registava qualquer evento cardio ou cerebrovascular anterior, bem como patologia psiquiátrica prévia. Foram excluídos hábitos alcoólicos e abuso de drogas. Foram excetuados quaisquer antecedentes familiares de demência/depressão major. Relativamente a factos recentes, à exceção da viuvez, não se reconheceram outras alterações do ambiente familiar, sendo que o agregado é constituído atualmente pela paciente, filha, genro e duas netas (18 e 12 anos), com os quais a paciente mantém boa relação, denotando-se, porém, a saída recente da neta mais velha que ingressou no ensino superior em Lisboa.

Atendendo à aparente deterioração cognitiva valorizada pela cuidadora, bem como pela presença de 
6 - HABILIDADE CONSTRUTIVA (1 ponto por cada cópia correcta.) Deve copiar um desenho. Dois pentágonos parcialmente sobrepostos; cada um deles deve ficar com 5 lados, dois dos quais intersectados: Não valorizar tremor ou rotação.

DESENHO

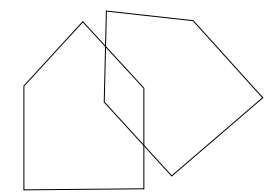

CÓPIA

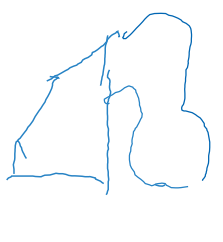

Figura 1. Representação da prova (Mini Mental State Examination - MMSE) da paciente aquando da primeira avaliação, na subcategoria do desenho.

fatores de risco cardiovasculares, foi solicitado estudo analítico (hemograma, glicemia, calcemia, ionograma sérico, função hepática, renal, tiroideia, vitamina B12, ácido fólico e teste sorológico para a sífilis) e imagiológico estrutural [tomografia computorizada (TAC) crânio-encefálica], segundo as recomendações nacionais vigentes na abordagem de alterações cognitivas. ${ }^{1}$ Foram colocadas como hipóteses mais prováveis de diagnóstico: quadro depressivo com declínio cognitivo ou processo demencial inicial com sintomas depressivos.

A doente e a cuidadora retornam pouco tempo depois, com TAC crânio-encefálica e estudo analítico sem alterações de relevo, tendo sido excluídas causas reversíveis/orgânicas que justificassem o respetivo quadro clínico (nomeadamente, quadros infecciosos, desregulação metabólica, desequilíbrios eletrolíticos ou síndroma de privação).

Pelo predomínio dos sintomas depressivos, a doente inicia prova terapêutica com sertralina $25 \mathrm{mg} /$ dia, com ajuste da dose para 50mg/dia após 15 dias. É agendada reavaliação em consulta e solicitado controlo analítico pelo risco acrescido de hiponatremia no idoso. Regressa após três meses, com melhoria significativa do humor, tendo retomado as suas atividades de lazer. A filha refere melhoria do desempenho funcional e menos compromisso da memória anterógrada. Atualmente mantém terapêutica, com estabilidade do quadro sintomático e cognitivo. MMSE atual: 26, sem défice cognitivo, denotando-se a execução perfeita da tarefa 6 (Figura 2) correspondente à habilidade construtiva, muito diferente do seu desempenho na primeira avaliação (Figura 1). Pelas características es- peciais que o mesmo caso imprime e pelas possíveis evoluções já descritas na literatura (evolução para possível demência), foi mantido o seguimento cuidado e periódico da doente.

\section{COMENTÁRIO}

A depressão no idoso representa uma entidade clínica ainda subdiagnosticada e tratada inadequadamente em cuidados de saúde. Cerca de $10 \%$ dos idosos que recorrem aos cuidados de saúde primários apresentam depressão clinicamente significativa. Uma meta-análise de 2010 indica que apenas metade dos casos são reconhecidos pelo médico de família, sendo que apenas um em cada cinco recebe tratamento eficaz. ${ }^{4,6-7}$

Perante um quadro semelhante ao supracitado é fundamental enfatizar o que há de diferente na realidade biopsicossocial do idoso, congregando aspetos sociais, psicológicos e orgânicos como fatores necessários para produzir e manter um quadro depressivo. ${ }^{6-7}$

Do ponto de vista vivencial, o idoso está numa situação de perdas continuadas: a privação de status ocupacional e económico, o declínio físico continuado, maior morbilidade e incapacidade funcional crescente. A falta de motivação, a indecisão, o negativismo, a lentidão psicomotora, o défice de atenção e a interferência cognitiva causada pelas preocupações quotidianas são alguns dos sintomas associados a este quadro de depressão na população geriátrica. ${ }^{7}$

Entre os principais fatores de risco para a depressão no idoso incluem-se: o género feminino, isolamento social, viuvez/divórcio, baixas condições socioeconómicas, comorbilidades, dor não controlada, insónia, insuficiência funcional, disfunção cognitiva, polimedicação e perturbações do sono. São ainda considerados fatores precipitantes de risco para depressão no idoso: luto recente, mudança de habitação/institucionalização, eventos de vida adversos (separação familiar, doença de familiar/cônjuge), episódios de depressão prévios, abuso de álcool e tipo de personalidade pré mórbida. ${ }^{7-9}$

Este quadro clínico torna-se ainda mais difícil de descortinar perante a sobreposição de aspetos que poderão estar presentes quer na depressão do tipo 
reativo, quer de causa secundária ou de origem endógena. Pelas condições frequentemente presentes no idoso, a depressão pode ser do tipo reativo (luto), de cariz secundário (devido a condição orgânica) ou de início endógeno (se presente personalidade pré mórbida ou episódios de depressão aquando adulto jovem). ${ }^{7,9}$

Ainda nos idosos e com relativa frequência, a tristeza é frequentemente mascarada por problemas somáticos. Este facto pode protelar o diagnósti-

6 - HABILIDADE CONSTRUTIVA (1 ponto por cada cópia correcta.) Deve copiar um desenho. Dois pentágonos parcialmente sobrepostos; cada um deles deve ficar com 5 lados, dois dos quais intersectados: Não valorizar tremor ou rotação.

DESENHO

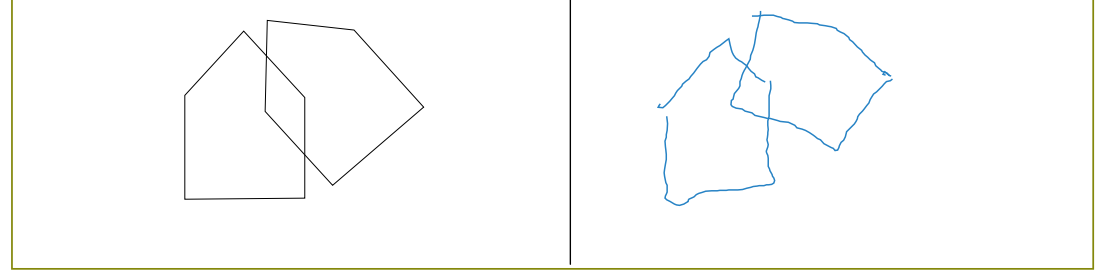

Figura 2. Desempenho da mesma utente na subcategoria do desenho do Mini Mental State Examination - MMSE após reavaliação e introdução de terapêutica antidepressiva. co e, consequentemente, o tratamen-

to, especialmente se existe patologia médica concomitante. Entre as morbilidades mais prevalentes depois dos 65 anos de idade e intrinsecamente relacionadas com sintomas depressivos destacam-se: as patologias do foro osteoarticular, a diabetes, a hipertensão arterial, a doença cardíaca, a perda auditiva e da visão, bem como a incontinência urinária. Vários estudos identificaram uma relação independente e robusta entre sintomas depressivos e a dor física crónica. Nos adultos mais velhos, a dor osteoarticular foi a mais comumente relacionada com a depressão. ${ }^{10}$ Perante estes sintomas dissimulados, o prestador de saúde deve considerar outros indícios como a fadiga persistente sem tradução orgânica, os distúrbios do sono, a dor crónica e ainda sintomas físicos medicamente inexplicáveis. ${ }^{10}$

A colheita de uma história clínica e exame físico cuidados são igualmente essenciais sempre que há suspeita de depressão/défice cognitivo/demência no idoso, permitindo fazer exclusão de causas secundárias, que podem ser diversas. Os exames laboratoriais devem incluir hemograma, ionograma sérico (para despiste de alterações eletrolíticas), glicose em jejum (para exclusão de diabetes e hipoglicemias em jejum, inclusive), função renal, hepática e tiroideia, vitamina B12 e folatos, cálcio e serologias para sífilis (nível de evidência C, grau de recomendação I). ${ }^{1,11}$ A Academia Americana de Neurologia, por exemplo, apenas recomenda o rastreio da deficiência de vitamina B12 e hipotiroidismo em doentes com suspeita de demência. Existem ainda estudos que aconselham a triagem da neurosífilis e VIH apenas se houver elevado grau de suspeição clínica. ${ }^{12}$ Apesar do estudo laboratorial se encontrar en- tre as orientações nacionais e guidelines internacionais, não há dados claros para apoiar ou refutar a necessidade de análises "seriadas" nestes casos (baixa relação custo-eficácia). ${ }^{13}$ A avaliação integral do doente deve merecer ainda a avaliação imagiológica estrutural, através de TAC cerebral, devido ao menor custo e maior disponibilidade deste exame. A ressonância magnética nuclear (RMN) é, no entanto, superior em termos de demonstração de lesões cerebrais, em particular alterações isquémicas. ${ }^{1}$ É de referir ainda a revisão da terapêutica habitual (e esporádica) do paciente idoso, mais vulnerável a interações medicamentosas e efeitos adversos que o adulto jovem. ${ }^{1,6}$

Outro aspeto a valorizar é a presença simultânea de eventual deterioração cognitiva. Sabe-se que a depressão, por si só, produz défice mnemónico, especialmente após os 40 anos de idade, podendo ser confundido com um quadro inicial de demência. A demência, por sua vez, define-se como o desenvolvimento de défices cognitivos múltiplos que incluem obrigatoriamente um compromisso da memória recente e, pelo menos, mais uma perturbação cognitiva (afasia, apraxia, agnosia ou perturbação na capacidade executiva). Estes devem ser suficientemente graves para terem repercussão funcional e representar um declínio em relação a um nível prévio de funcionamento e ocorrer na ausência de síndroma confusional (delirium). Os critérios da Classificação Internacional de Doenças (CID-10) são mais restritos, nomeadamente porque exigem que os défices cognitivos estejam presentes há pelo menos seis meses e requerem uma alteração no pensamento abstrato.

Esta constelação de sintomas do foro depressivo 
associada a alterações cognitivas pode produzir alterações aquando da aplicação de testes de avaliação cognitiva, especialmente nas categorias de atenção/cálculo e linguagem (especificamente o desenho) do MMSE. Face à falta de interesse do idoso deprimido é normal que ocorra alguma desatenção, menor destreza e algum desinvestimento na realização de tarefas, justificando-se a possibilidade de falsos positivos nos testes de screening (como é o caso da utente na primeira avaliação, ilustrado pela Figura 1). ${ }^{11-13}$ Por outro lado, exames neurocognitivos podem ser normais em caso de demência incipiente, principalmente em indivíduos com elevada escolaridade. Tal facto deve-se à capacidade superior de desempenho destes indivíduos, mesmo que já tenham ocorrido declínio de funções cognitivas. $^{14}$

Face a esta ambiguidade de resultados, a escala de avaliação da depressão geriátrica (GDS) ${ }^{15}$ é um instrumento importante e decisivo no diagnóstico diferencial. Esta escala existe em versão extensa (30 questões) e resumida (15 questões). Aborda sentimentos e comportamentos que decorreram na última semana, sendo que as respostas são dicotómicas (sim/não). Admite-se a presença de quadro depressivo perante uma pontuação final superior a cinco pontos.

Percebe-se, assim, que queixas de memória não implicam a existência de processos neurodegenerativos propriamente ditos. Sabe-se também que a autoperceção de comprometimento mnemónico na vida diária tende a estar preservada em idosos saudáveis, elemento este particularmente decisivo na diferenciação entre o patológico e o fisiológico. Nestes exemplos em que a queixa parte sobretudo do próprio e não tanto dos cuidadores e família, o declínio ocorre de modo discreto, acometendo principalmente a memória operacional sem que haja perturbação no quotidiano e implicações na autonomia do idoso. ${ }^{14}$

Em muitos casos, o quadro de deterioração cognitiva associado a sintomas depressivos pode ser estabilizado ou regredir apenas com tratamento antidepressivo. O tratamento psicofarmacológico da depressão no idoso depende essencialmente do perfil do paciente. Em grande parte dos casos, os inibidores seletivos da recaptação de serotonina (SSRIS) são considerados o tratamento de primeira linha, principalmente pela sua maior segurança e tolerabilidade.$^{1,16-18}$ Constituem a pri- meira escolha, sobretudo a sertralina, o citalopram e o escitalopram. Tal facto deve-se à longa semivida e menor interferência com o c-P450 destes fármacos. A mirtazapina pode ser também uma boa opção nos idosos com excessiva magreza, anorexia e apetite diminuído. A trazodona, por sua vez, será uma escolha plausível em idosos com perturbação do sono, fruindo-se do seu efeito antidepressivo e sedativo em simultâneo. Outros exemplos, como a venlafaxina e a duloxetina, poderão ser equacionados, à exceção dos indivíduos com hipertensão arterial pelo seu efeito nocivo. Em geral, os antidepressivos tricíclicos deverão ser evitados devido aos efeitos adversos frequentes na população idosa, atendendo ao seu efeito anticolinérgico. ${ }^{16-18}$

Sob a mesma linha de orientação é recomendada monoterapia a fim de minimizar efeitos colaterais e interações medicamentosas. Dever-se-á começar devagar e avançar lentamente (start low and go slow). A dose inicial deve ser ajustada para metade da dose habitual, com potencial ajuste até doses máximas, sendo que o tratamento a longo prazo (12-24 meses) é recomendável para prevenir a recorrência/recaída. ${ }^{16-18}$

Ao longo das consultas é pertinente questionar claramente sobre adesão à terapêutica (muitas vezes não cumprida por priorização das demais patologias orgânicas) e averiguar efeitos secundários. De forma a facilitar a complicance terapêutica, é importante reforçar positivamente pequenos progressos. ${ }^{6,19}$

As reações de privação/descontinuação são comuns na população geriátrica, sobretudo se o tratamento for suspenso abruptamente no caso dos SSRIs. O risco de hiponatremia no idoso é de considerar se este estiver medicado com sertralina. De considerar ainda o risco de prolongamento QT com o citalopram, que revelou uma associação dependente da dose deste efeito no eletrocardiograma. Paralelamente foram analisados dados de notificações espontâneas de prolongamento do intervalo QT e arritmia ventricular, incluindo torsades de pointes, recomendando-se $20 \mathrm{mg}$ como dose máxima diária no idoso. ${ }^{20}$

A psicoterapia parece ser outra alternativa eficaz, idealmente quando combinada com farmacoterapia e, sobretudo, em casos de depressão moderada a grave. Acredita-se que a depressão com mais sintomas somáticos responda menos à psicoterapia do que à medicação, embora a literatura relacionada seja escassa. ${ }^{21}$ 
A atividade física, quando regular e bem estruturada com o paciente/cuidador, pode também contribuir para a minimização do quadro depressivo, devendo por isso ser fomentado entre os idosos, independentemente da sua condição física. Tal intervenção oferece a oportunidade de envolvimento psicossocial, elevação da autoestima, estímulo cognitivo e efeito positivo acrescido perante outras morbilidades coexistentes. ${ }^{6}$

Vale ressaltar ainda que nem tudo é tão linear e previsível na depressão geriátrica quanto no adulto jovem. Isto porque, nem todos os défices observados são reversíveis com o tratamento adequado da depressão. Embora isso ocorra em parte dos casos, não deve ser considerada regra geral. ${ }^{14} \mathrm{Em}$ muitos dos estudos realizados, parte dos pacientes inicialmente diagnosticados como deprimidos apresentaram posteriormente verdadeiros sinais de demência, mesmo após melhoria inicial com o uso dos antidepressivos. ${ }^{11-14}$ Nesta condição é permitido considerar que o idoso com quadro depressivo estará mais comprometido que os seus pares não deprimidos. ${ }^{14}$ Nos casos em que se verifique declínio gradual, manutenção do quadro clínico com a terapêutica recomendada ou recidiva após melhoria, será oportuna a referenciação do caso para a consulta de neurologia/psicogeriatria.

Tomando este relato de caso como um dos muitos com que nos deparamos em consulta, importa considerar toda a marcha diagnóstica a fim de decifrar esta entidade tão particular no idoso.

A presença de sintomas somáticos inespecíficos e a própria condição médica concomitante, muitas das vezes debilitante, podem facilmente ocultar indícios de depressão, fazendo com que dificilmente seja diagnosticada pelo profissional de saúde.

Outra das barreiras ao tratamento eficaz tem a ver com o próprio idoso, que muitas das vezes parece resistir ao diagnóstico da depressão e atribuir os seus sintomas físicos à “idade”. Parte destes doentes têm um conhecimento limitado sobre esta patologia, construindo uma atitude estigmatizante face à depressão.

Adita-se também a linha ténue entre a demência e a depressão no idoso, cujas fronteiras movediças dificultam o rigor do seu diagnóstico. Este paradigma torna-se ainda mais complexo tendo em conta o comportamento atípico da depressão na terceira idade com respostas terapêuticas diversas ao tratamento dirigido, não se excluindo o risco de desenvolver demência no futuro, mesmo após melhoria da sintomatologia com terapêutica antidepressiva.

\section{CONCLUSÃO}

A depressão não é uma consequência natural do envelhecimento. A maioria dos idosos que recorre aos cuidados de saúde primários prioriza as queixas somáticas em detrimento do sentimento de tristeza que facilmente é reprimido. Por outro lado, poder-se-á estar a tratar pseudodemências em virtude de alterações cognitivas, potencialmente reversíveis com tratamento antidepressivo/psicoterapia.

Face a esta dubiedade torna-se essencial que o médico de família explore toda a sintomatologia inespecífica que traz o idoso à consulta, averiguando proativamente sinais e sintomas junto da família e seus cuidadores. A Escala de Depressão Geriátrica, instrumento criado especificamente para o diagnóstico da depressão em idosos e já validado na versão portuguesa, representa uma mais-valia para o médico de família perante quadros neurocognitivos mais inespecíficos.

Assim se entende que a terceira idade não é sinónimo de fragilidade, devendo o idoso ser "capacitado" pelos profissionais de saúde, família, entidades comunitárias e seus cuidadores. Todos eles serão peças decisivas para que se possa construir e sustentar o verdadeiro sentido do envelhecimento ativo, atenuando, desta forma, a extensão desta problemática do foro mental na população geriátrica.

\section{REFERÊNCIAS BIBLIOGRÁFICAS}

1. Direção-Geral da Saúde. Abordagem terapêutica das alterações cognitivas: norma da Direção-Geral da Saúde n. 0 053/2011, de 27/12/2011. Lisboa: DGS; 2011.

2. World Health Organization, Alzheimer's Disease International. Dementia: a public health priority. Geneva: WHO; 2012 [cited 2015 Sep 28]. ISBN 9789241564458 . Available from: http://apps.who.int/iris/bitstream/10665/75263/1/9789241564458_eng.pdf

3. Alzheimer's Association. 2014Alzheimer's disease facts and figures. Alzheimers Dement. 2014;10(2):e47-92.

4. Lin JH, Huang MW, Wang DW, Chen YM, Lin CS, Tang YJ, et al. Late-life depression and quality of life in a geriatric evaluation and management unit: an exploratory study. BMC Geriatr. 2014;14:77.

5. Morgado J, Rocha CS, Maruta C, Guerreiro M, Martins IP. Novos valores normativos do Mini Mental State Examination [New normative values of Mini Mental State Examination]. Sinapse. 2009;9(2):10-6.

6. Group Health. Dementia and cognitive impairment: diagnosis and treatment guideline. Group Health; December 2012. Available from: 
https://www.ghc.org/all-sites/guidelines/dementia.pdf

7. Park $M$, Unützer J. Geriatric depression in primary care. Psychiatr Clin North Am. 2011;34(2):469-87.

8. Cole MG, Dendukuri N. Risk factors for depression among elderly community subjects: a systematic review and meta-analysis. Am J Psychiatry. 2003;160(6):1147-56.

9. Vink D, Aartsen MJ, Schoevers RA. Risk factors for anxiety and depression in the elderly: a review. J Affect Disord. 2008;106(1-2):29-44.

10. Bisschop MI, Kriegsman DM, Beekman AT, Deeg DJ. Chronic diseases and depression: the modifying role of psychosocial resources. Soc Sci Med. 2004;59(4):721-33.

11. Birrer RB, Vemuri SP. Depression in later life: a diagnostic and therapeutic challenge. Am Fam Physician. 2004;69(10):2375-82.

12. Knopman DS, DeKosky ST, Cummings JL, Chui H, Corey-Bloom J, Relkin N, et al. Practice parameter: diagnosis of dementia (an evidencebased review). Report of the Quality Standards Subcommittee of the American Academy of Neurology. Neurology. 2001;56(9):1143-53.

13. Weytingh MD, Bossuyt PM, van Crevel H. Reversible dementia: more than $10 \%$ or less than $1 \%$ ? A quantitative review. J Neurol. 1995;242(7): 466-71.

14. Garcia A, Passos A, Campo AT, Pinheiro E, Barroso F, Coutinho G, et al. A depressão e o processo de envelhecimento [The depression and the aging process]. Cien Cognição. 2006;7:111-21. Portuguese

15. Yesavage JA, Brink TL, Rose TL, Lum O, Huang V, Adey M, et al. Development and validation of a geriatric depression screening scale: a preliminary report. J Psychiatr Res. 1982-1983;17(1):37-49.
16. Wiese BS. Geriatric depression: the use of antidepressants in the elderly. BCMJ. 2011;53(7):341-7.

17. Alexopoulos GS. Pharmacotherapy for late-life depression. J Clin Psychiatry. 2011;72(1):e04.

18. Bao Y, Post EP, Ten TR, Schackman BR, Bruce ML. Achieving effective antidepressant pharmacotherapy in primary care: the role of depression care management in treating late-life depression. J Am Geriatr Soc. 2009;57(5):895-900.

19. Voils $\mathrm{Cl}$, Steffens DC, Flint EP, Bosworth HB. Social support and locus of control as predictors of adherence to antidepressant medication in an elderly population. Am J Geriatr Psychiatry. 2005;13(2):157-65.

20. INFARMED. Citalopram - Risco de prolongamento do intervalo QT: circular informativa n. ${ }^{2}$ 230/CD, de 09/11/2011. Lisboa: INFARMED; 2011.

21. Gould RL, Coulson MC, Howard RJ. Cognitive behavioral therapy for depression in older people: a meta-analysis and meta-regression of randomized controlled trials. J Am Geriatr Soc. 2012;60(10):1817-30.

\section{CONFLITO DE INTERESSES}

As autoras declaram não ter conflitos de interesses.

\section{ENDEREÇO PARA CORRESPONDÊNCIA}

Joana Pinto Carneiro

E-mail: medjoanacarneiro@gmail.com

Recebido em 05-07-2015

Aceite para publicação em 20-03-2016

\section{ABSTRACT}

\section{DISTINGUISHING DEMENTIA FROM DEPRESSION IN THE ELDERLY: A CASE REPORT}

Background: Memory problems in the elderly are a frequent reason for consultation in primary health care. This common complaint can have different causes with different outcomes, emphasizing the need for early diagnosis and timely management. The following case illustrates how family doctors can approach this problem and manage the diagnosis and treatment of memory problems.

Case description: An 82-year old female patient widowed for 2 years and living with her daughter, who is her primary caregiver, came with her daughter to a medical appointment. The daughter complained that her mother was more apathetic, sadder, and sleepier in the last few months. She had been showing less interest in housework and did not want to leave the house as she formerly did. She had repetitive speech. Recently, she had experienced memory lapses and seemed to be slowing down. During the clinic visit, she told the doctor about her difficulty in paying for groceries, in recognizing acquaintances' names, and in recognizing familiar objects. She had been disoriented at times, getting lost more than once in the street. The possible diagnoses considered were depressive disorder with cognitive impairment or dementia with depressive symptoms. The patient began treatment with sertraline, showing significant improvement in mood, performance and cognitive function, without signs of a cognitive deficit.

Comment: Depression is not a natural consequence of old age. Many elderly people put physical complaints before feelings of sadness in primary care consultations. Pseudodementia may be potentially reversible with antidepressants and psychotherapy.

Keywords: Dementia; Cognitive Impairment; Depression; Elderly. 